Pavel Szobi

\title{
Lizenz- und Gestattungsproduktion westdeutscher Unternehmen in der ČSSR und der DDR
}

DOI 10.1515/jbwg-2017-0017

\begin{abstract}
The article deals with economic relations between the Federal Republic of Germany, German Democratic Republic and Czechoslovakia during the Cold War. Using the example of licensed production, its aim is to illustrate that in spite of ideological boundaries, business relations between West and East flourished in the period of the 1970s and 1980s. The author characterizes institutional conditions for this cooperation, names individual cooperation attempts, and uses the example of the well-known German brand Nivea as a symbol of the West and an example of a successful cooperation. The article reveals the intensive activities of West German companies and their investments in the GDR and Czechoslovakia long before 1989 and shows the potential of analyzing the German-German and the European transformation after 1989 more under the perspective of continuities and discontinuities.
\end{abstract}

JEL-Codes: N 34, N 44, N 64, N 74, N 84, O 14, O 31, O 33, P 33, P 36

Keywords: Tschechoslowakei, Deutsche Demokratische Republik, Lizenzproduktion, Gestattungsproduktion, Kosmetikindustrie, Czechoslovakia, German Democratic Republic, licensed production, authorized production, cosmetics industry, Beiersdorf

„,Eine Dame aus der Schweiz ist da. Wir müssen sie gut unterbringen‘, sorgt sich Ivana und führt mich in die Oase ihres Heims mit dem Gebot der Reinlichkeit und Ästhetik à la Schöner Wohnen. Im Badezimmer stehen wie Schutzengel des Hauses farbige Produkte westlicher Marken für die Körperpflege: Nivea-Creme, Schwarzkopf-Shampoo, JanaLotion. ,Mein Mann hat sie mir vor acht Jahren aus Deutschland mitgebracht. Sie sind längst aufgebraucht, ich fülle sie nach mit einheimischen Substanzen. Ich habe es gerne schön.` Ivana hat Potemkinsche Dörfer gebaut, sie ist zur Anbeterin fremder Götter geworden. Ist ästhetischer Genuss ein Menschenrecht? Mittelosteuropa vermutet unter dem

Pavel Szobi (Dr.), European University Institute, Department of History and Civilization, Via Bolognese 156, 50139 Florence, Italy, E-Mail: pavel.szobi@eui.eu 
Hochglanzpapier Würde, Schönheit, Leben an sich. ,Wir haben die Welt nicht gesehen. Dafür ist alles für das Heim. ““1

Dieses Zitat verdeutlicht den hohen Wert, den eine Frau aus Prag gegenüber ihrer Schweizer Besucherin westdeutschen Markenprodukten beimaß: Nicht nur das eigentliche Produkt, auch die Verpackung assoziierten Verbraucher in einer Mangelwirtschaft mit dem Ideal des westlichen Konsums. Dessen waren sich die Akteure sozialistischer Staatsbetriebe bewusst und versuchten Wege zu finden, den Wunsch der Verbraucher nach Westwaren zu erfüllen. Der vorliegende Beitrag behandelt das hiermit eng verbundene Agieren westdeutscher Unternehmen auf dem osteuropäischen Markt in den 1970er und 1980er Jahren. Es werden die spezifischen Umstände des West-Ost-Handels unter den Bedingungen des Kalten Krieges anhand des Beispiels der Hamburger Beiersdorf AG herausgearbeitet, die in vielen Ostblockländern ihre Produkte nicht nur erfolgreich vermarktete, sondern sie dort auch produzieren ließ. Dies wird an der Lizenz- und Gestattungsproduktion von Nivea-Produkten in der Tschechoslowakischen Sozialistischen Republik (ČSSR) und der Deutschen Demokratischen Republik (DDR) dargestellt. Das Beispiel von Nivea und der Kosmetikindustrie wurde ausgewählt, da Kooperationsverträge zwischen der Bundesrepublik und dem Ostblock in anderen Zweigen - etwa im Maschinenbau oder der Metallurgie - im Alltagsleben der Bevölkerung keine wesentliche Rolle spielten.

Nivea-Creme wurde bereits in der Zwischenkriegszeit zu einem bekannten Markenprodukt, das Beiersdorf in Hamburg sowie in ausländischen Filialen herstellte. Nach der Teilung Deutschlands war das Produkt Nivea in der DDR und ebenso in anderen Volksrepubliken bei den älteren Generationen immer noch in Erinnerung, als es Ende der 1960er Jahre wieder auftauchte. Beiersdorf bot folglich Produkte an, die sowohl Symbole der Nostalgie als auch Symbole des mit Westdeutschland bzw. -europa verbundenen Konsums und Wohlstands waren. An diesem Beispiel kann deswegen auf mehreren Ebenen gezeigt werden, wie eng die Verflechtungen zwischen Ost und West gewesen sind.

Der Beitrag konzentriert sich vor allem auf die 1970er und 1980er Jahre. Abschließend wird noch der Transformationsprozess der 1990er Jahre einbezogen. Der Fokus auf die 1970er Jahre hat dabei einen wesentlichen Grund: Infolge der Ostpolitik wurde 1972 der Grundlagenvertrag zwischen beiden deutschen Staaten unterschrieben, im Jahr darauf kam es zur Unterzeichnung eines Staatsvertrags zwischen Prag und Bonn. Dieser Rahmenvertrag wurde zwei Jahre später um

1 I. Brežná, Der Geruch von Freiheit und Nivea, in: der Freitag, 19.11.2009, http://www.freitag.de/ autoren/der-freitag/der-geruch-von-freiheit-und-nivea, 25.06.2016. 
das sogenannte Abkommen über die weitere Entwicklung der wirtschaftlichen, industriellen und technischen Zusammenarbeit erweitert. Während sich die Beziehungen zwischen der BRD und DDR graduell entwickelten und Anfang der 1980er Jahre vor allem durch den Strauß-Kredit beeinflusst wurden, forcierte die Regierung von Helmut Kohl keine intensivere Zusammenarbeit mit der ČSSR. Eine größere Rolle für die Entwicklung der wirtschaftlichen Kooperation spielte stattdessen der Privatsektor.

Dieses Thema wurde von tschechischen bzw. slowakischen Historikern bislang nur oberflächlich bearbeitet. Es existieren lediglich Studien zu einigen Teilproblemen der wirtschaftlichen West-Ost-Beziehungen nach 1945, während die Historiographie im deutschsprachigen Raum bereits deutlich detailliertere Betrachtungen hervorgebracht hat. Jenseits der Konzentration auf die deutschdeutschen Beziehungen ${ }^{2}$ widmen diese sich auch den Verbindungen zu anderen europäischen Ländern oder Regionen. ${ }^{3}$ Für den vorliegenden Beitrag relevant sind unter anderem die Arbeiten von Rainer Gries, insbesondere seine Analyse der Marken Nivea und Florena. ${ }^{4}$ In der tschechischen Geschichtsschreibung sind vor allem die Arbeiten von Jindřich Dejmek, ${ }^{5}$ Ivan Jakubec, ${ }^{6}$ Radko Břach, ${ }^{7}$

2 Aspekte der deutsch-deutschen Wirtschaftsbeziehungen werden u.a. beschrieben bei $A$. Steiner, Von Plan zu Plan. Eine Wirtschaftsgeschichte der DDR, München 2004; S. Wolle, Die heile Welt der Diktatur. Alltag und Herrschaft in der DDR 1949-1989, Berlin 2013; P.E. Fäßler, Durch den „Eisernen Vorhang“. Die deutsch-deutschen Wirtschaftsbeziehungen 1949-1969, Köln 2006.

3 Siehe z.B. J. Hecker-Stampehl, Nordeuropa und die beiden deutschen Staaten 1949-1989. Aspekte einer Beziehungsgeschichte im Zeichen des Kalten Krieges, Leipzig/Berlin 2007; E. Bischof, Honeckers Handschlag. Beziehungen Schweiz-DDR 1960-1990. Demokratie oder Diktatur, Bern 2010; K. Behling, Hightech-Schmuggler im Wirtschaftskrieg. Wie die DDR das Embargo des Westens unterlief, Berlin 2007.

4 Vgl. R. Gries, Produkte als Medien. Kulturgeschichte der Produktkommunikation in der Bundesrepublik und der DDR, Leipzig 2003. Der Nachteil der Studie besteht darin, dass dem Autor kein Zutritt zum Florena-Archiv gewährt wurde.

5 Vgl. J. Dejmek, Československo, jeho sousedé a velmoci ve XX. století (1918 až 1992). Vybrané kapitoly z dějin československé zahraniční politiky, Prag 2002.

6 I. Jakubec, Schlupflöcher im „Eisernen Vorhang“. Tschechoslowakisch-deutsche Verkehrspolitik im Kalten Krieg. Die Eisenbahn und Elbeschiffahrt 1945-1989, Stuttgart 2006; Ders., Československo-západoněmecké obchodně- politické a dopravněpolitické vztahy v období 19491967, in: Acta Oeconomica Pragensia 3, 2005, S. 190-209; Ders., Československo-západoněmecké obchodněpolitické a dopravněpolitické vztahy v období 1968-1989, in: Acta Oeconomica Pragensia 1, 2008, S. 38-52.

7 Vgl. $R$. Břach, Smlouva o vzájemných vztazích mezi ČSSR a SRN z roku 1973. Od prvních rozhovorů po ratifikaci smlouvy. Studie, Prag 1994; Ders., Die Bedeutung des Prager Vertrags von 1973 für die deutsche Ostpolitik, in: J.K. Hoench/H. Lemberg (Hg.), Begegnung und Kon- 
und Tomáš Vilímek ${ }^{8}$ von Bedeutung. Im Rahmen der Deutsch-Tschechischen Historikerkommission erscheinen regelmäßig Tagungsbände mit neuen Beiträgen zu diesem Thema. ${ }^{9}$ Auch liegen amerikanische Studien etwa von Jonathan Zatlin, Randall Newnham oder Ernest Plock vor. ${ }^{10}$ Besonders zu erwähnen sind auch die Arbeiten des britischen Historikers Gareth Dale zur Wirtschaftsverflechtung zwischen DDR und BRD. ${ }^{11}$ In diesen Publikationen wird die institutionelle Entwicklung der Wirtschaftsbeziehungen eingehend analysiert. Dagegen wird die Lizenz- und Gestattungsproduktion nur als kleiner Teil der Zusammenarbeit erwähnt. Dies überrascht insofern, als dass diese Kooperation nicht nur wirtschaftlich wichtig war, sondern auch für die Verbraucher in der DDR und ČSSR einen deutlichen Qualitätswandel im Warenangebot bedeutete. Die Beschäftigung mit der Lizenz- und Gestattungsproduktion gibt folglich auch Aufschluss über die Alltags- und Konsumwelt des Ostblocks und seine ideellen Bezüge. Außerdem lässt sich mit ihr die Frage beantworten, inwiefern die Kooperation zur Bewältigung der Transformationsprobleme der ostdeutschen und tschechischen/slowakischen Betriebe nach 1989 beitrug.

\section{Der institutionelle Rahmen für die Wirtschaftskontakte zwischen Bonn und Prag}

Nach der Gründung der Bundesrepublik Deutschland im Herbst 1949 bestanden zwischen den ehemaligen westlichen Besatzungszonen und Osteuropa - durch den Marshallplan und die Eingliederung der osteuropäischen Länder in die Organisation des Rates für gegenseitige Wirtschaftshilfe (RGW) - kaum Beziehun-

flikt. Schlaglichter auf das Verhältnis von Tschechen, Slowaken und Deutschen 1815-1989, Bonn 2001, S. 285-304.

8 Zum Thema dieser Studie vgl. z.B. T. Vilímek, Mladoboleslavská Škodovka v období normalizace (1969-1989), in: Ders. (Hg.), Česká společnost v 70. a 80. letech: sociální a ekonomické aspekty, Prag 2012, S. 63-175.

9 Siehe z.B. C. Buchheim/E. Ivaničková/K. Kaiserová/V. Zimmermann (Hg.), Die Tschechoslowakei und die beiden deutschen Staaten, Essen 2010.

10 Vgl. J.R. Zatlin, The Currency of Socialism. Money and Political Culture in East Germany, Cambridge 2007; E.D. Plock, East German-West German Relations and the Fall of the GDR, Boulder/San Francisco/London 1986; R.E. Newnham, Deutsche Mark Diplomacy. Positive Economic Sanctions in German-Russian Relations, University Park (PA) 2002.

11 G. Dale, Between State Capitalism and Globalization. The Collapse of the East German Economy, Oxford 2004. 
gen. ${ }^{12}$ In der ersten Hälfte der 1950er Jahre strebte der RGW das Ziel an, fast den ganzen Außenhandel unter den Mitgliedsstaaten und vor allem mit der Sowjetunion zu organisieren. Obwohl im Rahmen der Hallstein-Doktrin die Bundesrepublik keine diplomatischen Vertretungen in den RGW-Ländern außer der UdSSR hatte, war die Bonner Regierung in Handelsfragen viel flexibler. Denjenigen Unternehmen, die Investitionspläne für die RGW-Staaten hatten, wurde tendenziell geraten, sich nicht von der politischen Entwicklung abschrecken zu lassen. ${ }^{13}$ So reisten etwa im Fall der Tschechoslowakei westdeutsche Diplomaten und Geschäftsmänner nach Prag und verfassten hierüber Berichte. In der persönlichen Korrespondenz des ehemaligen deutschen Botschafters in London, Hans Schlange-Schöningen, ist zum Beispiel folgender Bericht eines Mülheimer Fabrikanten erhalten, der im Jahr 1959 eine Reise in die Tschechoslowakei unternommen hatte:

„Von meiner Reise aus Prag bin ich zurück. Die Behandlung war sehr zuvorkommend. [...] Die Leute müssen exportieren, wenn sie nicht hungern wollen, denn ihre Landwirtschaft ist unter dem Hund. [...] Ein erheblicher Teil der Lebensmittel muß eingeführt werden [...]. Neue moderne Kreuzritter müssen wieder ausziehen, um [...] wenigstens einen Teil unseres Bodens wieder unter unsere Kontrolle zu bekommen. [...] Wir investieren schließlich in anderen Gebieten auch Kapital, warum nicht auch im Osten? Der Standpunkt mit den Polen und Tschechen überhaupt keinen Handel zu treiben und diese völlig zu ignorieren, ist grundfalsch.“14

Ein paar Jahre später waren die Berichte westdeutscher Unternehmer schon positiver gestimmt. Sie gaben ihrer Überraschung über einigermaßen gute Lebensbedingungen Ausdruck, wobei sie aber das unzureichende Warenangebot in den Geschäften und die schlechte Infrastruktur bemängelten. ${ }^{15}$

Im März 1963 unterzeichneten die Regierungen der ČSSR und der BRD ein Protokoll über den Warenaustausch zwischen beiden Ländern, mit dem das Warenverkehrsabkommen von 1961 verlängert wurde. Zwei Jahre später kam es wegen unterschiedlicher Ansichten bezüglich der Berliner Klausel und des Münchner Abkommens zu keiner Verlängerung. Beide Länder hielten sich je-

12 Vgl. z.B. K. Kaplan, Československo v RVHP: 1949-1956, Prag 1995; L.K. Metcalf, Council of Mutual Economic Assistance. The Failure of Reform, New York 1977, S. 18-47.

13 K. Rudolph, Wirtschaftsdiplomatie im Kalten Krieg. Die Ostpolitik der westdeutschen Großindustrie 1945-1991, Frankfurt a.M. 2005, S. 212.

14 Brief von E. Wienkoop an Hans Schlange-Schöningen, 16.02.1959, Bundesarchiv Berlin (BArch) N 1071/15.

15 Bericht über deutsch-tschechische Besprechungen am 24./25.05.1963 in Prag sowie über Reiseeindrücke in der ČSSR, BArch B 102/135271. 
doch an die Regeln des Protokolls von 1963, das de facto bis in die zweite Hälfte der 1960er Jahre gültig war. Bei der damit verbundenen, überaus seltenen Gelegenheiten für den Austausch zwischen westdeutschen und tschechoslowakischen Regierungs- und Handelseliten wurde aber deutlich, dass sich Tschechen und Slowaken eine intensivere Zusammenarbeit wünschten. So wurde 1963 dem Leiter des Ost-Ausschusses der deutschen Wirtschaft, Otto Wolff von Amerongen, bei einer solchen Gelegenheit auf der Brünner Messe angedeutet, man habe in der Tschechoslowakei Interesse an wesentlich mehr Handel und Kooperation - und zwar in ähnlicher Form, wie sie von der BRD bereits mit Polen, Ungarn, Rumänien oder Bulgarien realisiert wurde. ${ }^{16}$ Allerdings verkomplizierten politische Hindernisse die Gespräche; erst 1967 kam es zur Eröffnung der westdeutschen Handelsvertretung. ${ }^{17}$ Jenseits politischer Unstimmigkeiten lag einer der Hauptgründe dieser im Vergleich zu Ungarn, Polen und Bulgarien langsamen Entwicklung darin, dass das tschechoslowakische Exportprofil nicht wie die meisten Ostblockländer Agrarprodukte als Handelsware vorsah. Vielmehr sollten industrielle Fertigwaren nach Westeuropa exportiert werden. Das Jahr 1968 und die folgende Re-Zentralisierung der politischen und wirtschaftlichen Ordnung in der ČSSR zeitgenössisch als Normalisierung bezeichnet - machten die Hoffnung auf eine Intensivierung der Handelskontakte aber zunichte. Andere Ostblockländer nutzten derweil die Bereitschaft westeuropäischer Länder, private Mittel in die sozialistische Industrie zu investieren. ${ }^{18}$ Im Gegensatz dazu überwog in den 1970er Jahren unter der Föderalregierung des Premierministers Lubomír Štrougal eine konservative Finanzpolitik. Sein autarkiepolitischer Kurs wurde aktiv von seinen Schlüsselministern durchgesetzt, vor allem von Außenhandelsminister Andrej Barčák, Finanzminister Leopold Lér oder dem Vorsitzenden der Tschechoslowakischen Staatsbank, Stanislav Potáč. Damit wandte sich die politische Elite des Landes gegen zahlreiche Devisenanträge tschechoslowakischer Betriebe, die sich für moderne Produktionsanlagen und Lizenzkäufe Westkredite erhofften.

Als sich 1973 das Verhältnis zwischen ČSSR und BRD durch den Vertrag über gemeinsame Beziehungen normalisierte, ${ }^{19}$ wurde seitens der tschechoslowakischen Staatsbetriebe die Forderung nach einem umgehenden Ausbau der Zusammenarbeit mit Westdeutschland laut. In der BRD erhoffte man sich von den neuen Wirtschaftsbeziehungen mit der Tschechoslowakei, den größer geworde-

16 Rudolph, Wirtschaftsdiplomatie, S. 209.

17 Vgl. Jakubec, Československo-západoněmecké obchodněpolitické, S. 194 f.

18 Vor allem die Nachbarländer Ungarn und Polen waren bei der Suche nach Kontakten zu westeuropäischen Firmen besonders aktiv.

19 Vgl. Břach, Smlouva. 
nen Einfluss italienischer und französischer Firmen im Ostblock auszugleichen. Vor allem handelte es sich dabei um Investitionen im Maschinenbau und in der Kfz-Industrie..$^{20}$ Die Manager des deutschen Automobilkonzerns BMW verlangten sogar vom Bundeswirtschaftsministerium, dass die Bundesregierung selbst die Interessen deutscher Fahrzeughersteller vertreten sollte, denn ,durch die Errichtung der FIAT- und RENAULT-Werke in einigen sozialistischen Ländern ist hier bereits nahezu jegliche Basis für einen aktiven Export der deutschen Kraftfahrzeugindustrie verloren gegangen“. ${ }^{21}$ Obwohl beispielsweise Volkswagen bereits Ende der 1970er Jahre ein Projekt zum Einbau deutscher Motoren in die ŠkodaAutos mit den tschechoslowakischen Partnern aus der AZNP Mladá Boleslav (heutige Škoda) ausarbeitete, kam es vor 1989 nicht zu einer praktischen Umsetzung. Allerdings waren genau diese Kontakte zwischen Volkswagen und AZNP Mladá Boleslav in der Transformations- und Privatisierungsphase der Škoda-Werke fruchtbar, als es zur Modernisierung des Fahrzeug-Models Favorit kam. Nach 1989 wurde Škoda ein Teil des deutschen VW-Konzerns. Erfolglos blieben vor 1989 auch die Kooperationsgespräche der Tatra-Werke in Kopřivnice mit der Kölner Firma Kloeckner-Humblodt-Deutz AG, die sich 1978/79 um eine gemeinsame Herstellung von luftgekühlten Motoren für Lastkraftwagen bemüht hatten. ${ }^{22}$

Dies ist vor allem auf die Unfähigkeit der Akteure in der tschechoslowakischen Industrie zurückzuführen; sie wollten die Exporterlöse fertiger Industriewaren auf einem hohen Stand halten. Außerdem verschlechterten sich die Handelsbedingungen für den Export nach Westeuropa in den 1970er Jahren kontinuierlich. Gründe dafür waren die niedrige Arbeitseffektivität, die hohen Produktionskosten und steigende Preise für Rohstoffimporte. Diese Bedingungen verlangten eine pragmatische und offene Einstellung gegenüber ausländischen Investitionen. 1975 kam es deswegen zur Ratifizierung des Abkommens über die weitere Entwicklung der wirtschaftlichen, industriellen und technischen $\mathrm{Zu}$ sammenarbeit, das ein wichtiges Element für eine neue Phase der Kooperation

zwischen der BRD und der ČSSR darstellte. Einen wesentlichen Fortschritt brachte dabei die neu gegründete Deutsch-tschechoslowakische Kommission für Koope-

20 Für Informationen über die finanzielle Beteiligung von FIAT an dem Aufbau des Automobilwerks in Tyrnau vgl. den Bericht über die Dienstreise in die ČSSR vom 21.-23.06.1971, 30.06.1971, BArch B 102/78324.

21 Brief an den Staatssekretär des Bundesministeriums für Wirtschaft und Finanzen Dr. Schlöllhorn, Betr.: PKW-Export in die ČSSR - Ihr Schreiben vom 10.02.972, BArch B 102/78324.

22 Industrielle Kooperation khd/tatra, Brief des Herrn Brenning von Kloeckner-HumblodtDeutz AG für Herrn Moeldner aus dem Wirtschaftsministerium (BMWI), 12.03.1979, BArch B $102 / 204464$. 
ration mit sich, die erstmals im Herbst 1975 in Prag konferierte. ${ }^{23}$ Die Bedeutung der Kommission wurde dabei durch den gemeinsamen Vorsitz des Bundeswirtschaftsministers und des Vizepremiers der tschechoslowakischen föderalen Regierung verdeutlicht. Neben der Hauptkommission wurden spezialisierte Fachgruppen für einzelne Industriezweige gegründet. ${ }^{24}$ Diese institutionelle Basis verhalf zu neuen Kontakten zwischen den Regierungsvertretern und den Betriebsmanagements beider Länder und beschleunigte den Abschluss von Kooperationsverträgen.

In der zweiten Hälfte der 1970er Jahre wurden etwa 30 Kooperationsverträge ausgehandelt, hauptsächlich Lizenzverträge in der Chemie- und Eisenhüttenindustrie: Vacmetal (Hoesch) aus Dortmund investierte in den Bau und Betrieb von Vakuumanlagen für die Verarbeitung von flüssigem Stahl in Vítkovice, ThyssenRheinstahl installierte Entschwefelungsanlagen für Roheisen in Východoslovenské železiarne, Košice. Die Lizenzvergaben waren jedoch nicht einseitig: Vielmehr vergaben tschechoslowakische Firmen - ebenfalls vornehmlich aus der chemischen Industrie - auch Lizenzen an deutsche Unternehmen. So erhielt beispielsweise die Firma Dynamit Nobel eine Lizenz zur Herstellung des Metallbeizmittels Feropur. ${ }^{25}$ Die tschechoslowakische Regierung war allerdings mit der geringen Anzahl der Kooperationsverträge unzufrieden und drängte angesichts der vergleichsweise höheren Anzahl an Verträgen, die westdeutsche Firmen etwa in Polen oder Ungarn bereits ausgehandelt hatten, auf eine Steigerung der Vertragsabschlüsse. Dabei beachtete die tschechoslowakische Seite allerdings nicht die sinkende westdeutsche Konjunktur in den 1970er Jahren. Außerdem erfolgte die wirtschaftliche Annäherung der ČSSR an die Bundesrepublik vergleichsweise spät - die Regierungen in Budapest und Warschau hatten bereits in den 1960er Jahren entgegenkommende Gesetze für deutsche Investitionen und die Schaffung von Joint-Venture-Betrieben entwickelt. ${ }^{26}$ Die Hans Schwarzkopf $\mathrm{GmbH}$, die in Ungarn verschiedene Kosmetikprodukte herstellte, konnte im Rahmen dieser Kooperationen besonders erfolgreich agieren und schließlich im Jahre 1985 einen gemeinsamen Betrieb gründen - die Schwarzkopf Kozmetikai

23 Programmentwurf für die erste Tagung der deutsch-tschechoslowakischen Wirtschaftskommission in Prag, 3.09.1975, BArch B 102/240368.

24 Aktennotiz über die Besprechungen der Vorsitzenden der Fachgruppe Chemie und Energie vom 16.-18.12.1975 in Frankfurt a.M., 18.12.1975, BArch B 102/240367.

25 Übersicht der Kooperationsprojekte, September 1977, BArch B 102/240369; Kooperationsabkommen zwischen der Industrie der ČSSR und ausländischen Unternehmen (Messebeobachtung Brünn 1970), Rheinisch Westfälisches Wirtschaftsarchiv (RWWA) 175-13-2.

26 Interne Auskunft, 1.08.1977, betr. ČSSR-Klagen über Wirtschaftsbeziehungen, hier: Strougal gegenüber Botschafter Diesel vom 20.07.1977, BArch B 102/240369. 
Kft. ${ }^{27}$ Hierbei ist indes $\mathrm{zu}$ berücksichtigen, dass einige Projekte nicht realisiert werden durften, da sie gegen die Regeln der COCOM-Kommission für Technologietransfers verstoßen hätten - so zum Beispiel im Fall geplanter Kooperationen der Firmen Leit und Bosch. ${ }^{28}$

Zudem agierten die tschechoslowakischen Behörden und Firmen in der Kommunikation mit den westdeutschen Partnern mitunter unprofessionell. So berichtete Eckart Grams vom Ost-Ausschuss der deutschen Wirtschaft im April 1983: „In Brünn im letzten September wurde den deutschen Referenten ein kleiner, stickiger Saal mit uninteressierten, vor sich hindösenden Zuhörern zugemutet. “29

Obwohl hier der Eindruck entsteht, dass die eher langsame Entwicklung der institutionellen Koordinierung die wirtschaftliche Verkopplung deutscher und tschechoslowakischer Firmen einschränkte, so zeigen einige Beispiele auch eine intensive wirtschaftliche Zusammenarbeit: Große deutsche Konzerne wie Krupp oder Siemens warteten nicht auf den Segen der Bundesbehörden und waren in der Lage, eigeninitiativ Projekte in der ČSSR umzusetzen. Neben dem Maschinenbau etablierten sich in der Tschechoslowakei dabei vor allem Firmen aus der Lebensmittel- und Kosmetikindustrie. Und tschechoslowakische Betriebe überraschten die deutschen Partner mit der Fähigkeit, einen großen Anteil ihrer für den EG-Markt bestimmten Produktion vor allem in die Bundesreplik exportieren zu können. ${ }^{30}$

\section{Gestattungsproduktion in der DDR}

Lizenzverträge waren für westdeutsche und westeuropäische Unternehmen ein gangbarer Weg, im Binnenmarkt osteuropäischer Länder aktiv zu werden. Deswegen bemühten sich westeuropäische Firmen nicht nur in Ostmitteleuropa, sondern auch in der Sowjetunion um Verträge mit einheimischen Betrieben. In einigen Ostblockländern entwickelte sich die Zusammenarbeit dabei schneller

27 Geschäftsbericht 1984. Hans Schwarzkopf GmbH, Hamburg 1985, S. 19.

28 Dr. Bodo Bötther, Geschäftsführer des Zentralverbandes der Elektronischen Industrie e.V. an Bundesministerium für Wirtschaft, Herrn Min. Rat Dr. W. Jahnke, Betr.: Deutsch-tschechoslowakische gemischte Fachgruppe für Elektrotechnik und Elektronik, vom 10.04.1981, RWWA, F. 175-13-2.

29 Eckart Garms vom Ostausschuss an Joachim Wischermann, Leiter der Handelsförderungsstelle der Botschaft der Bundesrepublik Deutschland, Prag, vom 7.04.1983, RWWA, F. 175.12.4. 30 Aufzeichnung, Betr.: Verhandlungen EG-ČSSR über ein Textilselbstbeschränkungsabkommen, 5.05.1981, BArch B 102/270542. 
als in anderen. Vor allem nach dem Grundlagenvertrag 1972 steigerte sich die deutsch-deutsche Zusammenarbeit auf staatlicher und betrieblicher Ebene enorm. Während in den meisten Ländern klassische Lizenzverträge vereinbart wurden, handelten die DDR-Betriebe einige Verträge im Rahmen der sogenannten Gestattungsproduktion aus. ${ }^{31}$ Nach der Einschätzung von Alexander SchalckGolodkowski, Leiter des Bereichs Kommerzielle Koordinierung (KoKo) im ostdeutschen Außenhandelsministerium, waren es insbesondere westdeutsche Unternehmen, die sich an die DDR-Behörden wandten. ${ }^{32}$ Die konkrete Initiative sei dabei von dem Vorstandsvorsitzenden der Salamander AG, Franz Josef Dazert, ausgegangen. ${ }^{33}$

Die Gestattungsproduktion basierte darauf, dass ostdeutsche Betriebe genaue Produktionsregeln einzuhalten hatten, um die Qualität der westdeutschen Partner $\mathrm{zu}$ erzielen. Im Gegensatz zu Lizenzverträgen wurde nicht mit einer Lizenzgebühr, sondern mit einem Teil der produzierten Ware bezahlt. Der Großteil der in der DDR produzierten Westware wurde folglich an den westdeutschen Markt abgegeben. Der deutlich geringere Rest stand dem DDR-Binnenmarkt zur Verfügung. ${ }^{34}$ Die Gestattungsproduktion war insofern eine Win-Win-Situation für beide Seiten: Die westdeutschen Betriebe bekamen billige aber dennoch hochwertige Produkte, die für die Verbraucher in der BRD vermeintlich aus Westproduktion stammten. Und den DDR-Verbrauchern konnten umgekehrt begehrte Westprodukte angeboten werden, ohne die knappen Devisen bzw. Valutamark

31 Die Gestattungsproduktion ist nicht zu verwechseln mit der Beratungsproduktion, bei der Herstellungsmethoden mit einheimischen Mitteln übernommen wurden; siehe H. Weiß, Verbraucherpreise in der DDR. Wie stabil waren sie?, Schkeuditz 1998, S. 65.

32 Zur Rolle des Bereichs KoKo vgl. z.B. Deutscher Bundesstag (Hg.), Beschlußempfehlung und Bericht des 1. Untersuchungsausschusses nach Artikel 44 des Grundgesetztes. Der Bereich Kommerzielle Koordinierung und Alexander Schalck-Golodkowski. Werkzeuge des SED-Regimes. Textband, Bonn 1994. Die Aufgaben und Organisation der KoKo werden von Mathias Judt detailliert analysiert; vgl. M. Judt, KoKo - Mythos und Realität. Das Imperium des Alexander Schalck-Golodkowski, Berlin 2015.

33 A. Schalck-Golodkowski, Deutsch-deutsche Erinnerungen, Reinbek 2000, S. 189. Diese Interpretation ist überaus subjektiv. Schon in den 1960er Jahren befassten sich Staatsministerien der DDR mit der Idee, Lizenzen aus Westdeutschland zu erwerben; vgl. hierzu beispielsweise $B$. Ciesla, Eine sich selbst versorgende Konsumgesellschaft? Industrieller Fischfang, Fischverarbeitung und Fischerwarenkonsum in der DDR, in: T. Lindenberger (Hg.), Herrschaft und EigenSinn in der Diktatur. Studien zur Gesellschaftsgeschichte der DDR, Köln 1999, S. 205-233.

34 J. Roesler, Momente deutsch-deutscher Wirtschafts- und Sozialgeschichte 1945 bis 1990. Eine Analyse auf gleicher Augenhöhe, Leipzig 2006, S. 193 f. 
in der Staatskasse zu belasten. ${ }^{35}$ Viele Produkte aus der Gestattungsproduktion wurden wegen ihres vermeintlich westlichen Ursprungs allerdings nicht in den gewöhnlichen Kaufhallen oder Drogerien der DDR verkauft, sondern in den Devisengeschäften von Intershop oder Intertank. So wurden in diesen Läden beispielsweise erhebliche Mengen an Zigaretten westlicher Marken angeboten, von denen ca. 90 Prozent in der DDR hergestellt worden waren. ${ }^{36}$

Insgesamt wurden in der DDR etwa 100 westdeutsche Produkte in Gestattungsproduktion hergestellt, unter anderem von Firmen wie Beiersdorf, BAT, Bosch (Blaupunkt), Nestlé (Bärenmarke), Salamander, Schiesser, Triumph, Trumpf, Underberg, Varta oder Privileg (Quelle). ${ }^{37}$ In den 1970er und 1980er Jahren entwickelte sich die Gestattungsproduktion zu einem erkennbar wichtigen Bestandteil der ostdeutschen Wirtschaft. So wurden etwa 1987 in der DDR 4,6 Millionen Paar Schuhe für die Firma Salamander produziert. ${ }^{38}$ Die durchaus erhebliche Quantität der Gestattungsproduktion kann als Beweis für die Abhängigkeit der DDR-Wirtschaft von westlicher Hilfe interpretiert werden. ${ }^{39}$ Schalck-Golodkowski vertrat zwar die Ansicht, dass die westdeutschen Unternehmer sich sehr darum bemüht hätten, den DDR-Markt für sich zu gewinnen: „In manchen Branchen, wie dem hart umkämpften Lebensmittelmarkt, standen bundesdeutsche Unternehmer geradezu Schlange, um ins Geschäft zu kommen. “40 Die vielfältigen Kontakte waren aber zum gegenseitigen Vorteil.

Von der Tatsache, dass die in der DDR angebotenen Westwaren in großen Mengen in der DDR selbst produziert wurden, nahm die Bevölkerung zunächst wenig Notiz. Viele Konsumenten fühlten sich jedoch betrogen, wenn sie auf den

35 Die Gestattungsproduktion hat bei einem großen Teil der ehemaligen DDR-Elite einen schlechten Ruf und wird beispielsweise vom ehemaligen Staatssekretär Klaus Blessing stark kritisiert; vgl. K. Blessing, Die Schulden des Westens. Was hat die DDR zum Wohlstand der BRD beigetragen? Berlin 2010, S. $50 \mathrm{f}$.

36 Vgl. A. Volze, Devisengeschäfte der DDR. Genex und Intershop, in: Deutschland Archiv 11, 1991, S. 1145-1159, hier S. 1151; B. Ciesla/P. Poutrus, Food Supply in a Planned Economy. SED Nutrition Policy between Crisis Response and Popular Needs, in: K. Jarausch (Hg.), Dictatorship as Experience. Towards a Socio-Cultural History of the GDR, New York/Oxford 1999, S. 143-162, hier S. $145 \mathrm{f}$.

37 Vgl. P. Krewer, Geschäfte mit dem Klassenfeind. Die DDR im innerdeutschen Handel 19491989, Trier 2008, S. 241.

$38 \mathrm{Vgl}$. K. Marxen/G. Werle, Strafjustiz und DDR-Unrecht. Amtsmissbrauch und Korruption, Berlin 2002, S. 327.

39 Die Hausmitteilungen und Notizen von Partei- und Staatsorganen in der DDR deuten auf diese Abhängigkeit klar hin: vgl. z.B. SED-Hausmitteilung, Information zur Sicherung des NSW-Exportplanes 1982, S. 3, BArch DY 30/25068.

40 A. Schalck-Golodkowski, Deutsch-deutsche Erinnerungen, S. 190. 
tatsächlichen Herstellungsort - zum Beispiel bei genauerem Blick auf die Verpackung - aufmerksam wurden. Eingaben und Beschwerden dokumentieren diese Unzufriedenheit der Konsumenten. Sie wurden in der DDR-Gesellschaft als eine Form der „Mitbestimmung und Mitgestaltung“ genutzt. Der amerikanische Historiker Jonathan Zatlin ordnet sie als die einzige Möglichkeit ein, in einem Land ohne funktionierende Parteien, Verbände, Justiz und unabhängige Medien seine Meinung zu vertreten. ${ }^{41}$ So schrieb ein Soldat der Nationalen Volksarmee in Erfurt in seiner Eingabe, dass er eine Tafel der westdeutschen Alpenmilchschokolade Karina für 6,80 Ostmark gekauft hätte. Beim Öffnen der Packung stellte er fest, dass die Schokolade in der DDR hergestellt worden war. In seiner Eingabe erklärte er, die Schokolade schmecke ähnlich wie die Markenschokolade des VEB Thüringer Schokoladenwerk Saalfeld/Saale, die allerdings zum viel niedrigeren Preis von 3,85 Ostmark angeboten wurde. Welche Wichtigkeit diesen Eingaben beigemessen wurde, zeigt die Tatsache, dass der Generaldirektor des Schokoladenwerks, das 95 Prozent aller Tafelschokoladen in der DDR herstellte und 1.660 Mitarbeiter beschäftigte, angewiesen wurde, dem Soldaten eine Antwort zu schreiben. ${ }^{42}$ In diesem Schreiben erläuterte er, dass der hohe Preis der Karina-Schokolade durch die Lizenz und die einzuführenden Ingredienzen bedingt war. Die Frage, warum die Karina-Schokolade genauso wie die einheimische DDR-Schokolade aussah und schmeckte, wurde allerdings nicht beantwortet. ${ }^{43}$

Einer der letzten realisierten Verträge war der Vertrag für die Gestattungsproduktion der traditionsreichen Firma Florena in Waldheim, die 1852 unter dem Namen Waldheimer Parfümerie- und Toilettenseifenfabrik gegründet worden war. ${ }^{44}$ Seit 1955 wurde in Waldheim die beliebte Florena-Creme in einer Niveaähnlichen Blechdose produziert. ${ }^{45}$ Die Beiersdorf AG beanstandete diese Aufma-

41 J.R. Zatlin, Ausgaben und Eingaben. Das Petitionsrecht und der Untergang der DDR, in: ZfG 10, 1997, S. 902-917, hier S. 906.

42 Brief von J. G., 21.09.1985, BArch DY 30/25626; C. Jacobi, Der Schokoladenkönig. Das unglaubliche Leben des Hans Imhofff, München, Berlin 1997, S. 37.

43 Brief vom Generaldirektor des VEB Thüringer Schokoladenwerk Saalfeld/Saale, Helmut Bretschneider, 18. 10.1985, BArch DY 30/25626. Zur Schokoladenqualität gibt es viele anderen Eingaben, siehe z.B. die Sammlung RWWA 208-3-3.

44 A. Zschiesche/O. Errichiello, Florena - der Osten auf der Haut, in: Dies. (Hg.), Erfolgsgeheimnis Ost. Survival-Strategien der besten Marken - und was Manager daraus lernen können, Wiesbaden 2009, S. 124.

45 Ihre gute Qualität beruhte auf den technischen Herstellungsbedingungen in Waldheim. Bei dem Besuch einer Arbeitsgruppe der Kozmetika Bratislava waren die slowakischen Gäste vom hohen technischen Standard des Waldheimer Betriebs sehr beeindruckt; vgl. Správa zo zahraničnej služobnej cesty do NDR - závody VVB Chemisches Kombinat Militz - závod Zitza 
chung des Produktes. Im Jahr 1975 gelang es, eine Abgrenzungsvereinbarung mit Florena zu treffen: Die Creme durfte laut dieser Vereinbarung nur in sozialistischen Ländern in einem blauen Dosenunterteil mit weißer Beschriftung angeboten werden. Für Dosen, die zum Verkauf in anderen Ländern bestimmt waren, musste der DDR-Hersteller ein weißes Unterteil verwenden. ${ }^{46}$ Die Hamburger entschieden sich schließlich Ende der 1980er Jahre, dem Betrieb das Recht zur Herstellung von Nivea-Creme zu übertragen. Im Oktober 1989 gelangte die erste in Ostproduktion hergestellte Marge auf den Markt. Im Vergleich zur einheimischen und qualitativ vergleichbaren Florena-Creme, deren 60-Milliliter-Packung 1,95 Ostmark kostete, musste der DDR-Kunde für eine 75-Milliliter-Packung Nivea in spezialisierten Geschäften 5,50 Ostmark zahlen. ${ }^{47}$ Florena Waldheim plante, 4,5 Millionen Cremepackungen herzustellen. Als Gegenleistung mussten davon 1,5 Millionen Stück an die Beiersdorf AG und an den westdeutschen Markt abgegeben werden. Der Rest durfte sowohl in der DDR als auch im RGW-Raum verkauft werden. Angesichts des Devisenmangels der DDR ist es jedoch kaum überraschend, dass das Politbüro des ZK der SED auch den Verkauf ihres NiveaKontingents im nichtsozialistischen Ausland plante und zwar in der Größenordnung von einer Millionen Packungen im Wert von 0,6 Millionen Valutamark. ${ }^{48}$ Die DDR-Kunden, für die die neue Ware zu teuer war oder die sie in Geschäften wegen der geringen Menge nicht erhalten konnten, waren daher auf die 13,2 Millionen Einheiten Florena-Creme angewiesen..$^{49}$

\section{Lizenzproduktion in der ČSSR}

Wie für die DDR war es auch für die anderen RGW-Länder wichtig, sowohl wirtschaftliche als auch politische Beziehungen nach Westen zu knüpfen. In der Tschechoslowakei war diese Bedingung mit der Öffnung der Bonner Handels-

Zeitz a Florena Waldheim, Štátny archív Bratislava, Fond Kozmetika Bratislava, Operatívne porady 1975 , Karton 9.

46 Gries, Produkte, S. 550.

47 Information an G. Mittag vom 21.09.1987, S. 1, Barch DY 30/6980.

48 Anlage Nr. 3 zum Protokol Nr. 105 vom 23.09.1987, Betr.: Vorbereitung der Gestattungsproduktion von Nivea-Creme, Barch DY 30 J IV 2/3, 4164, S. 21; Beschluß des Sekretariats des ZK der SED vom 23.09.1987, Betr.: Vorbereitung der Gestattungsproduktion von Nivea-Creme, BArch DC 20/I/4 6102.

49 Information zur Angebots- und Preisgestaltung für die neue Gestattungsproduktion von Nivea-Creme, S. 1, Barch DY 30/6980. 
vertretung 1967 erfüllt. Während es für ostdeutsche Firmen typisch war, mit den Partnern aus der BRD Verträge über die Gestattungsproduktion auszuhandeln, handelte die Tschechoslowakei hauptsächlich reguläre Lizenzverträge aus.

In den Jahren 1971 und 1977 war Prag der Gastgeber der Hamburger Wirtschaftstage, an denen auch die Delegation des Hamburger Hafens teilnahm. Die Tschechoslowakei war der zweitwichtigste Partner für dessen Warentransit. ${ }^{50}$ Mehrere Hamburger Firmen signalisierten aufgrund der ohnehin schon bestehenden Kontakte ihr Interesse an Investitionen in der Tschechoslowakei - unter ihnen auch die Beiersdorf AG, die mit dem Konzern Fettindustrie Prag ins Gespräch kam. Dieser vereinte im Rahmen der Planwirtschaft fast die gesamte tschechoslowakische Kosmetikindustrie unter einem Dach. ${ }^{51}$ In der Fettindustrie waren wichtige Kosmetikstandorte unter anderem Severočeské tukové závody in Ústí nad Labem, Povltavské tukové závody in Nelahozeves, Závody Kosmo in Čáslav, Rakona in Rakovník, Závody Milo in Olomouc, Aroma in Prag und Vitana in Byšice.

Das slowakische Unternehmen Kozmetika Bratislava war für die Kosmetikindustrie in der Slowakei zuständig und bildete einen eigenen „Resortbetrieb“, war jedoch mit dem Konzern in Produktionsfragen und -aufgaben verbunden. Mehrere dieser Betriebe existierten unter verschiedenen Namen seit dem 19. Jahrhundert und wurden im Sinne der Effektivität und Lenkung der Fettindustrie Prag zugeordnet, wobei der Konzern selbst dem Landwirtschaftsministerium unterstellt war. ${ }^{2}$

Die Fettindustrie Prag nutzte die Dienstleistungen ihres Außenhandelsvermittlers AHB Polytechna, um konkrete Bedingungen über Lizenzvergaben auszuhandeln..$^{53}$ Dies wurde mit Mitarbeitern von Beiersdorf koordiniert, die konkrete Lizenzvorschläge an die Fettindustrie Prag einreichten. Beiersdorf wollte dem Konzern mehrere Produkte zur Lizenzproduktion anbieten, darunter Handund Sportcremes, Körpermilch oder Sonnenöle. Diese Initiative traf die Fettin-

50 Stand der deutsch-tschechoslowakischen Wirtschaftsbeziehungen, 22. 09.1977, BArch B 102/240369. Vgl. zur Bedeutung der tschechoslowakischen Präsenz in diesem Hafen vor allem Jakubec, Schlupflöcher.

51 Die Wirtschaftseinheit Fettindustrie wurde bis 1965 von einer Generaldirektion geleitet, dann wurde sie in einen Trust und 1974 in einen Konzern umgewandelt.

52 Vgl. Statut výrobní hospodářské jednotky Tukový průmysl, trust podniků, Prag, Státní oblastní archiv (SOA) Praha, Fond Povltavské tukové závody, Statut národního podniku, Karton 3.

53 Aktennotiz über Verhandlungen mit den Herren Direktor Filip und Dr. Malý von der Generaldirektion der Fettindustrie, Prag, 4.-6.11.1968 in Hamburg, Konzernarchiv Henkel, Fond Schwarzkopf GmbH, K-Akte ČSSR I, DE000006785. 
dustrie-Betriebe jedoch unvorbereitet: Es stellte sich heraus, dass die Staatsbetriebe gar nicht über die notwendigen Kapazitäten und Technologien verfügten, um simultan mehrere Lizenzprodukte herzustellen. Für den Kooperationsstart schien die Kozmetika Bratislava noch am ehesten geeignet, da das Werk seit 1965 über neue Produktionsanlagen aus Italien verfügte. Das Aushandlungsverfahren bis zur Unterzeichnung kam dann nur relativ langsam voran und dauerte von Juni bis Oktober $1967 .^{54}$

Bei Produktionsanlauf der Nivea-Creme musste auch der Import von Dosen für die Cremes aus Österreich oder Italien organisiert werden, weil in der ČSSR keine geeigneten Muster zur Verfügung standen. Bei der Suche nach geeigneten Zulieferern, die die Blechdosen produzieren konnten, kam ein Kontakt mit dem Betrieb Strojobal in Gablonz zustande, der auf Verpackungsherstellung spezialisiert war. Kurioserweise konnte ein Mitarbeiter in einer Besprechung ein Blechdosenmuster präsentieren, das vor dem Krieg in Strojobal für die Nivea-Herstellung genutzt worden war. ${ }^{55}$ Wegen des Devisenmangels gab es zwischen der Beiersdorf AG und Kozmetika Bratislava eine Absprache darüber, dass die Slowaken Dosen aus einheimischer Produktion benutzten durften. ${ }^{56}$ Es kam deshalb gelegentlich zu geringen Abweichungen vom deutschen Original.

Die Direktion der Fettindustrie entschied kurz nach den Vertragsverhandlungen, für die Nivea-Produkte einen völlig neuen Betrieb zu gründen: Astrid Prag. ${ }^{57}$ Der Standort wurde Ende 1968 eröffnet und nahm mit der Unterzeichnung des aktualisierten Lizenzvertrags im März 1969 die Produktion auf. ${ }^{58}$ Die Lizenzkosten waren mit Beiersdorf folgendermaßen geregelt: Der deutsche Partner erhielt eine Lizenz von fünf Prozent auf den Netto-Verkaufserlös der Vertragsprodukte abzüglich der vom Umsatz abhängenden Steuern. Die Lizenzbasis war der in der BRD geltende Fabrikabgabepreis, der sich aus dem Verbraucherpreis

54 Brief von Eric Knapp an Hans Schwarzkopf vom 12.11.1966, Konzernarchiv Henkel, Fond Schwarzkopf GmbH, K-Akte ČSSR I, DE000006785.

55 Hauskurier. Die Zeitschrift der Beiersdorfer 84, September 1973, S. 7.

56 Brief vom tschechischen Landwirtschaftsministerium an Beiersdorf vom 5.03.1969, Beiersdorf AG, Corporate \& Brand History Archive, Fach 413, Akte Tschechoslowakei - Lizenzverträge.

57 Aktennotiz über Verhandlungen mit den Herren Direktor Filip und Dr. Malý von der Generaldirektion der Fettindustrie, Prag, 4.-6.11.1968 in Hamburg, Konzernarchiv Henkel, Schwarzkopf GmbH, K-Akte ČSSR I, DE000006785; Brief vom tschechischen Landwirtschaftsministerium an Beiersdorf vom 5.03.1969, Beiersdorf AG, Corporate \& Brand History Archive, Fach 413, Akte Tschechoslowakei - Lizenzverträge.

58 Unterschriebene Originale der Verträge mit Astrid und Milo, Konzernarchiv Henkel, Schwarzkopf GmbH K-Akte ČSSR I, DE000006785. 
abzüglich der üblichen Handelsspannen ergab..$^{59}$ In den folgenden Jahren verlangte der AHB Polytechna zwar günstigere Lizenzsteuern von den westdeutschen Partnern, da die tschechoslowakische Regierung den Druck in der Deviseneinsparung erhöhte. In Hamburg wies man jedoch darauf hin, dass den Betrieben in der ČSSR bereits sehr günstige Bedingungen gewährt würden zum Beispiel die kostenlose Vergabe von Maschinen, Reparaturen oder Materialien für Werbezwecke. ${ }^{60}$

Wie erwartet war die Nachfrage nach den neuen Beiersdorf-Erzeugnissen enorm. Die Milo-Werke erhielten sogar Beschwerden von Verbrauchern, weil die im Fernsehen beworbenen Produkte in den Geschäften gar nicht verfügbar waren. ${ }^{61}$ In Hamburg bemängelte man, dass die farbigen Werbeanzeigen in den tschechoslowakischen Frauenmagazinen einen verblassten und unästhetischen Eindruck machten. ${ }^{62} \mathrm{Zu}$ Kritik kam es auch in einem anderen Fall: Beiersdorf bewarb im berühmten Kurort Karlsbad ihre Bonbonmarke Hustinetten, da sich hier viele Kurgäste aus der Bundesrepublik aufhielten - sogar der „HustinettenBär“, eine beliebte Werbefigur der Firma, war in der Stadt zu sehen. In der Tschechoslowakei wurden Hustinetten aber gar nicht verkauft. Die Werbung bewegte eine tschechische Frau aus dem benachbarten Ort Loket dazu, einen Brief an die Zentrale in Hamburg zu schreiben:

„Auf diese Reklame freue ich mich nicht alleine, sondern auch meine Tochter und meine zwei Enkelkinder. [...] Trotzdem wissen wir nicht, was das für Bonbons oder Tabletten sind [...]. Aber der Reklame nach sind sie bestimmt gut und helfen."63

Die westdeutschen Partner kontrollierten regelmäßig die Qualität der hergestellten Lizenzwaren. Für die Betriebsbesuche wurden die Anlagen gründlich gereinigt und die Angestellten trugen weiße Arbeitsmäntel, die sonst nur zu wichtigen Anlässen wie dem Umzug zum 1. Mai angezogen wurden. ${ }^{64}$ Die Erfüllung der Qualitätsstandards zahlte sich aus: In Archivdokumenten von Schwarzkopf ebenfalls eine Vertragsfirma der Fettindustrie Prag - sind Briefe von zufriede-

59 Beiersdorf AG, Corporate \& Brand History Archive, Fach 413, Akte Tschechoslowakei Lizenzverträge.

60 Brief von Herrn Mynarek aus der Auslandsabteilung der Frima Schwarzkopf vom 21.06.1971, Konzernarchiv Henkel, Fond Schwarzkopf GmbH, K-Akte ČSSR I, DE000006785.

61 Zpráva e služební cesty do NSR k firmě Schwarzkopf Hamburg ve dnech 15.-20.12.1970, SOA Olomouc, Fond Milo, s. p. Olomouc, Folge-Nr. 14, Karton 61.

62 Ebda.

63 Hauskurier. Die Zeitschrift der Beiersdorfer 81, Mai 1973, S. 7.

64 Zápis z porady vedení konané dne 30.09.1985, SOA Praha, Fond PTZ, Zápisy z porad vedení, Karton 12. 
nen Verbrauchern aus der Tschechoslowakei und auch von westdeutschen Touristen erhalten. ${ }^{65}$

Da in der tschechoslowakischen Wirtschaft Devisenengpässe zu einem immer größeren Problem wurden, sollten die Lizenzprodukte wie in der DDR reexportiert werden. Dies war jedoch laut den Lizenzverträgen nur auf dem RGWMarkt möglich. Durch neue Lizenzverträge von Beiersdorf mit anderen osteuropäischen Ländern wurde dies noch weiter eingeschränkt - hier wurden die RGW-Staaten mit ihren Westprodukten gar Konkurrenten. Bereits 1978 musste Astrid deswegen den Export nach Rumänien, in die DDR und die UdSSR einstellen. ${ }^{66}$ Dafür wurde den tschechoslowakischen Partnern von Beiersdorf aber erlaubt, die Lizenzprodukte an Drittländer zu verkaufen - und zwar nach Libyen, Syrien, in den Irak sowie in den Iran. ${ }^{67}$

Die Fettindustrie Prag fand einen Ausweg in neuen Kooperationsverträgen mit Beiersdorf, die der Gestattungsproduktion, die für die DDR typisch war, ähnelten: Der Konzernbetrieb Povltavské tukové závody stellte für deutsche Partner die Kaloderma- und Nivea-Seifen her. Ein Teil der Nivea-Seifen wurde dann für den Binnenmarkt freigegeben. Insgesamt produzierte Povltavské tukové závody im Jahre 1973 etwa 3.700 Tonnen Seife. Für 1986 waren bereits 7.000 Tonnen geplant, davon fast 6.000 Tonnen Toilettenseifen. Von der Gesamtmenge der Toilettenseifen entfielen mehr als 1.060 Tonnen auf die Nivea-Seifen, hiervon waren aber nur 55 Tonnen für den tschechoslowakischen Binnenhandel bestimmt. Der Rest musste exportiert werden, um die nötigen Devisenerlöse zu erzielen. ${ }^{68}$ Die steigende Produktion rief jedoch ein neues Problem hervor: Diese Produktionsmenge der Konzernfirmen übte einen enormen Druck auf die generelle Arbeitseffektivität aus, die mit Gastarbeiterverträgen aufgefangen werden musste. So arbeiteten 1986 wiederholt polnische Arbeiter in Betrieben des Konzerns Fettindustrie, vor allem bei Severočeské tukové závody. ${ }^{69}$ Auch wurde die

65 Brief von Frau Köhler aus Solingen an die Firmenleitung von Schwarzkopf vom 11.01.1986, Konzernarchiv Henkel, Fond Schwarzkopf GmbH, K-Akte ČSSR I, DE000006786.

66 Brief der Firma Schwarzkopf an die Fettindustrie Prag vom 27.07.1978, Konzernarchiv Henkel, Fond Schwarzkopf GmbH, K-Akte ČSSR I, DE000006785.

67 Dies geschah jedoch erst Ende der 1980er Jahre; vgl. Zpráva ze zahraniční cesty do NSR za účelem zabezpečení licenční výroby na základě uzavřených smluv s fou H. Schwarzkopf, Hamburg a fou Beiersdorf, Hamburg, Termín cesty 6.-11.03.1988, 31.03.1988, SOA Olomouc, Fond Milo, s. p. Olomouc, Folge-Nr. 14, Karton 61.

68 Zápisy z porad vedení podniku 1987, SOA Praha, Fond PTZ, Karton 12; 8. PLP. Návrh plánu na rok 1986. Mýdla, SOA Praha, Fond PTZ, Karton 55.

69 Zápis z 5. Rady ředitelů koncernu Tukový průmysl, Praha, která se konala dne 22.05.1986 v 9,30 hod. v Praze 1, Opletalova 4, SOA Praha, Fond PTZ, Porada ředitelů 1983, Karton 16; 
Möglichkeit geprüft, für eine längere Laufzeit Arbeiter aus Vietnam einzustellen; dies wurde jedoch als administrativ nicht durchsetzbar abgelehnt. ${ }^{70}$ Die fehlenden Arbeitskräfte wurden auch in der einheimischen Bevölkerung gesucht, sehr oft arbeiteten am Wochenende Arbeiter aus der Landwirtschaft als Hilfskräfte in den Betrieben. ${ }^{71}$

\section{Fazit: Florena Waldheim und die Fettindustrie Prag nach 1989}

Ab 1989 geriet die Gestattungs- und Lizenzproduktion in den ehemaligen Ostblockländern in die Krise, später wurde sie gar überflüssig. Importe aus dem Westen beendeten die Dominanz der Lizenzwaren. So wurde Milo Olomouc im Oktober 1990 darauf hingewiesen, dass die Verpackungsqualität zu verbessern sei, um mit den importierten Waren konkurrieren zu können. ${ }^{72}$ Im Lauf des Jahres 1990 erwogen westdeutsche Firmen, in der Tschechoslowakei eine neue Form der Zusammenarbeit zu entwickeln, da Fettindustrie Prag Ende Juni 1990 aufgelöst wurde und sich die Lizenzverträge nun auf sieben unabhängig gewordene Staatsbetriebe verteilten. ${ }^{73}$ In der unübersichtlichen Situation der Transformation (Rechtsunsicherheit, unbekannte Besitzerverhältnisse im Laufe der Privatisierung, Kommunikationsprobleme) entschieden die Unternehmen in Deutschland schließlich, die Lizenzverträge 1992 bzw. 1993 auslaufen zu lassen und eigene Filialniederlassungen in Tschechien und in der Slowakei zu gründen. Von den ursprünglichen Betrieben durchliefen die Astrid AG und Setuza AG (Severočeské tukové závody) eine erfolgreiche Transformation; ihr Produktportfolio verkleinerte sich allerdings enorm und beinhaltete keine Lizenzprodukte mehr. Aus Kozmetika Bratislava gingen zwei Firmen hervor - die Palma AG und die AB Kozmetika AG. Da viele Produktionsanlagen während der 1970er und 1980er mit

Pokyny $\mathrm{k}$ řešení otázek spojených se zaměstnáním polských dělníků v Československu, 23.04.1964, SOA v Litoměřicích, pracoviště Most, Fond STZ, pův. č. 438, časový rozsah 19481991, Karton 3.

70 Zápis z porady vedení konané dne 23.11.1981, SOA Praha, Fond PTZ, Zápisy z porad vedení, Karton 12.

71 Zápis z porady vedení konané dne 4.05.1986, SOA Praha, Fond PTZ, Zápisy porady vedení, Karton 12.

72 Besuchsbericht, 24.10.1990, SOA Olomouc, Fond Milo, s. p. Olomouc, Folge-Nr. 14, Karton 61.

73 Zpráva ze zahraniční služební cesty, vykonané do SRN ve dnech 7.-17.10.1990, SOA Olomouc, Fond Milo, s. p. Olomouc, Folge-Nr. 14, Karton 61. 
Importen aus der Bundesrepublik modernisiert worden waren, konnten die Firmen auch unter marktwirtschaftlichen Bedingungen konkurrenzfähig bleiben. Die Privatisierung von AB Kozmetika mündete jedoch in zahlreiche Schwierigkeiten, die letztendlich zur Produktionseinstellung führten.

In der ehemaligen DDR vollzog sich eine sehr spezifische Entwicklung, die nicht nur mit dem Übergang zur Marktwirtschaft, sondern auch mit der Wiedervereinigung Deutschlands verbunden war. Viele der ostdeutschen Betriebe überlebten diesen Übergang nicht - entweder konnten sie mit ihrer Produktion mit den westdeutschen Firmen nicht konkurrieren oder sie wurden von diesen privatisiert und später oft liquidiert. Ausnahmen bilden zwei bereits erwähnte Betriebe: Florena Waldheim änderte ihren Namen in Florena Cosmetic GmbH. Die Firma konnte, dank der Zusammenarbeit mit Beiersdorf, überleben. Im Jahr 1998 exportierte sie ihre Produkte in über 35 Länder. 2002 wurde der Betrieb vollständig von der Beiersdorf AG übernommen und in Beiersdorf Manufacturing Waldheim $\mathrm{GmbH}$ umbenannt. Diese ist ein bis in die Gegenwart erfolgreiches Unternehmen mit ca. 300 Angestellten, das weiterhin Florena- und Nivea-Creme herstellt. $^{74}$

Die Stollwerck AG, die mehrere Lizenzen an das Thüringer Schokoladenwerk vergeben hatte, konnte sich nach dem Mauerfall mit den DDR-Partnern nicht über eine weitere Zusammenarbeit einigen. Deswegen ging der Saalfelder Standort im März 1990 eine Kooperation mit der Firma Alfred Ritter GmbH \& Co. KG ein. Der Cannstatter Partner übernahm den Betrieb in Saalfeld und es wurde die neue Markenschokolade Thürina eingeführt. Das Produkt war jedoch nur kurz auf dem Markt; nachdem die Währungs-, Wirtschafts- und Sozialunion in Kraft gesetzt wurde, war die Thürina-Schokolade gegenüber den Westwaren nicht länger konkurrenzfähig. Die Zusammenarbeit mit Ritter wurde aufgelöst. Vor dem Schicksal anderer DDR-Betriebe wurden die Saalfelder jedoch durch das persönliche Engagement des Stollwerck-Aufsichtsratsvorsitzenden Hans Imhoff gerettet. Er handelte mit der Treuhandanstalt spezifische Bedingungen aus und verpflichtete sich, jährlich Waren im Wert von 280 Millionen Mark abzunehmen. Das Schokoladenwerk wurde wieder zum größten Schokoladenproduzenten in den neuen Bundesländern. ${ }^{75}$

74 Vgl. D. Wurzel, Beiersdorf will Florena stärken - Produkte der Ost-Marke verlassen auch Waldheimer Werk, in: Leipziger Volkszeitung, 5.04.2017, http://www.lvz.de/Region/Doebeln/ Beiersdorf-will-Florena-staerken-Produkte-der-Ost-Marke-verlassen-auch-Waldheimer-Werk, 9.04.2017.

75 Jakobi, Der Schokoladenkönig, S. 39. 


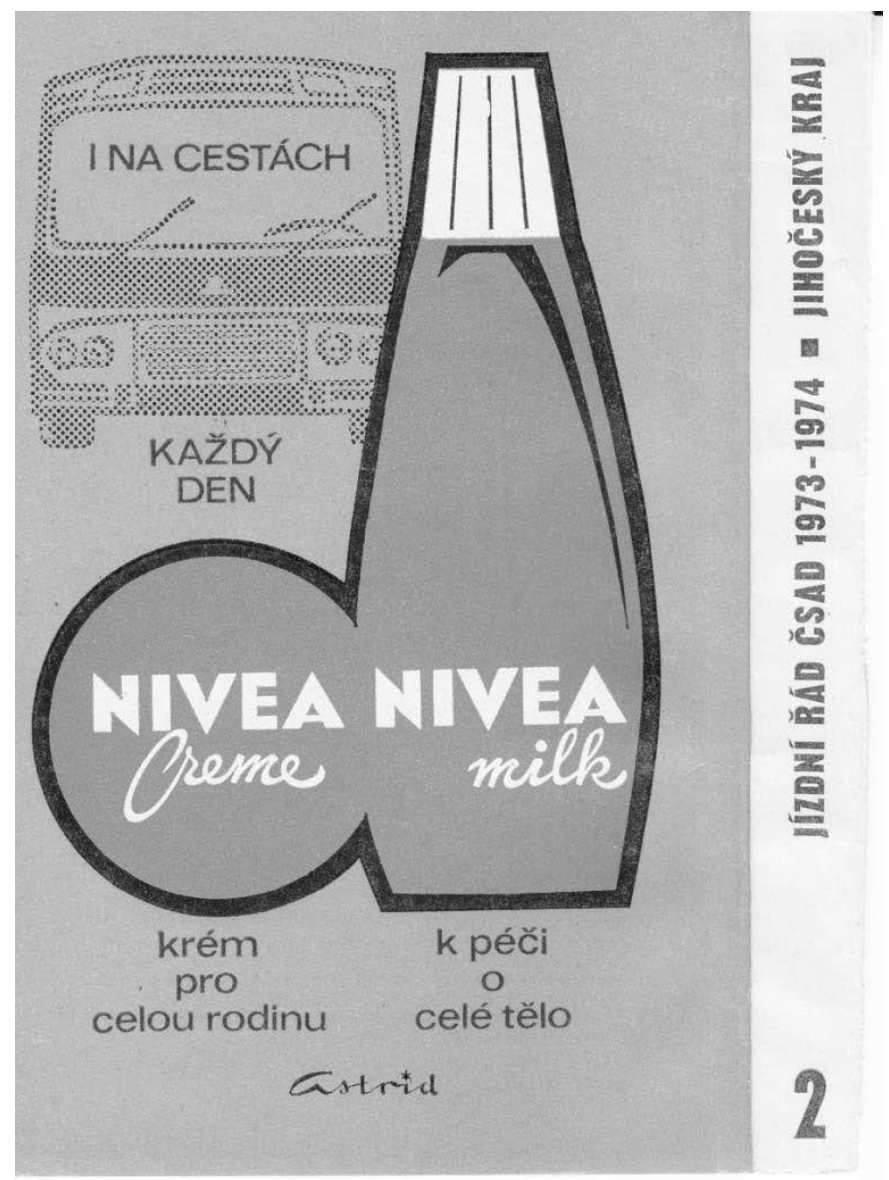

Abb. 1: Eine Werbung von Nivea auf der Vorderseite eines Busfahrplanes im Südböhmischen Bezirk für die Jahre 1973 bis 1974. Im unteren Teil ist die Marke des Herstellers Astrid deutlich sichtbar. Quelle: Beiersdorf AG, Corporate \& Brand History Archive, Bestand Plakate NI-

VEA/CSSR/1960er-80er.

Die vorgestellten Beispiele der Gestattungs- und Lizenzproduktion in der DDR und der ČSSR zeigen, dass die Kosmetikbetriebe in beiden Ländern infolge der Kooperation mit westdeutschen Firmen die Qualität des Warenangebots am Binnenmarkt erhöhten. Die Wirtschaftlichkeit der Produktion blieb jedoch problematisch. Importierte Rohstoffe wurden in den 1970 Jahren teurer; zudem gab es Schwierigkeiten mit geeigneten Verpackungen. Die Lizenzprodukte wurden zwar beworben, blieben aber in vielen Fällen Mangelware, da vor allem die Produkte der Gestattungsproduktion an Beiersdorf geliefert wurden und damit 
dem Binnenmarkt und den dort geweckten Bedürfnissen nicht zur Verfügung standen. Produktionsverträge und langjährige Zusammenarbeit waren jedoch wichtig, um die Probleme des Transformationsprozesses $\mathrm{zu}$ überwinden. Die Staatsbetriebe mit Lizenz- und Gestattungsproduktion verfügten deswegen bereits über moderne Maschinen und Fertigungsstraßen, die ihnen im Vergleich $\mathrm{zu}$ anderen Staatsbetrieben einen gewissen Vorteil verschafften. In Gemeinschaft mit westlichen Kooperationspartnern und Kapital wurde die Transformation $\mathrm{zu}$ Privatfirmen in manchen Fällen deswegen erleichtert, wie es vor allem die ostdeutschen Beispiele zeigen.

Widmung: Der Beitrag wurde im Rahmen des Projektes "PanEur1970s: Looking West: the European Socialist regimes facing pan-European cooperation and the European Community" erarbeitet. Das Projekt wurde vom Europäischen Forschungsrat im Rahmen des EU-Programms für Forschung und Innovation Horizon 2020 unterstützt (Grantabkommen Nr. 669194).

\section{Bionote}

\section{Pavel Szobi}

(1983) holds an MA and Ph.D. in History from the Charles University in Prague and works as research associate in the PanEur1970s Project at the European University Institute in Florence. He specializes in history of Czechoslovakia after 1945 and history of Eastern Bloc. 